\title{
Design Considerations for the Implementation of a Mobile IP Telephony System in a Nuclear Power Plant
}

\author{
J. García-Hernández¹, J. C. Velázquez- Hernández ${ }^{1}$, \\ C. F. García-Hernández ${ }^{1}$ and M. A. Vallejo-Alarcón ${ }^{2}$ \\ ${ }^{1}$ Electric Research Institute (IIE) \\ ${ }^{2}$ Federal Commission of Electricity (CFE) \\ Mexico
}

\section{Introduction}

IP telephony, also called voice over Internet protocol (VoIP), is rapidly becoming a familiar term and technology that is implementing in the enterprise, education, government organizations and industry. Mobile IP telephony is the new generation of communications networks that makes possible the convergence of voice and data over wireless local area networks (WLANs). This technology combines data networks with mobile technologies to support voice and data applications over a common integrated network. A key advantage of IP telephony is that it allows the transmission of voice signals from conventional telephones over an IP data network, being either a public network (Internet) or a private network (Intranet). Figure 1 shows a general IP telephony system. IP telephony is designed to replace the legacy TDM (time division multiplexing) technologies and networks by an IP-based data network. Digitized voice will be carried in IP data packets over a LAN and/or WAN network. A major aspect involved in a voice conversation using mobile IP telephony is the conversion of analog or digital voice signals from conventional phones to IP packets for further transmission either to a fixed or mobile phone, over an IP network. One of the most important recommendations that can be made is to pay close attention to the infrastructure

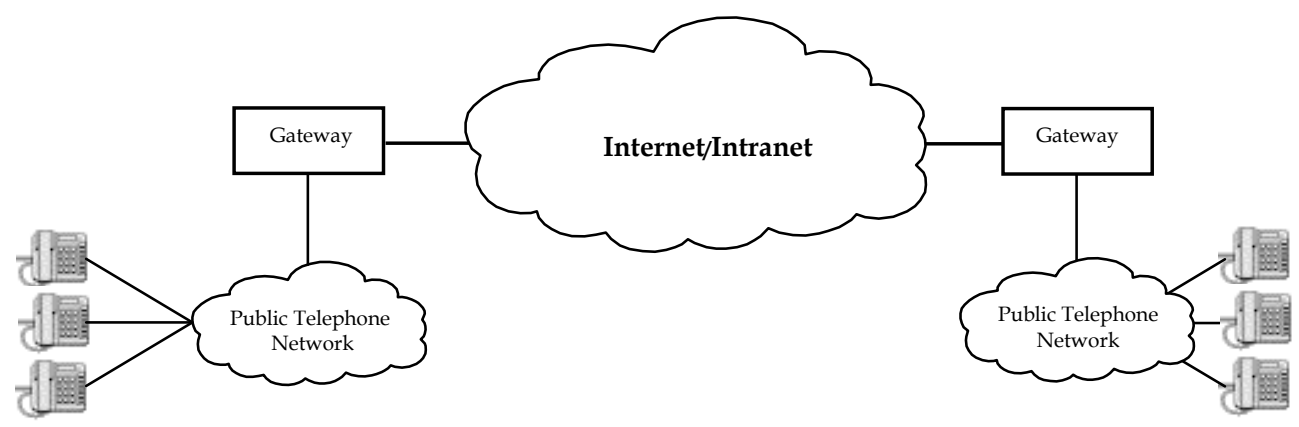

Fig. 1. General IP telephony system 
that the IP telephony network is built on. The design considerations must be solid otherwise; there will be ongoing quality issues until the network design issues are resolved. Even though IP telephony have made some vast reliability and quality improvements in recent years, customers and network designers still struggle with implementing the technology in a multivendor network. There are many reasons for this, such as interoperability issues and proprietary protocols. In addition, the use of new wireless technologies in nuclear power plants is growing fast. The WLAN technology based on the IEEE 802.11 standard has a very promising future for its use in nuclear power plants, due to features like mobility, reliability, security, scalability and compatibility with other technologies in order to provide new services such as voice over IP (VoIP) and IP video (Shankar, 2003).

In this work, the design considerations for the implementation of a mobile IP telephony system in a nuclear power plant based on national and international standards are presented, as well as, technical requirements that commercially available equipment must meet. In addition, this work gives an analysis of the most relevant wireless technologies currently available that can be implemented in nuclear power plants and also identifies nuclear regulatory guidelines, wireless networks standards, electromagnetic and radiofrequency interference standards. In the next sections, an analysis of the most relevant wireless technologies currently available that can be implemented in nuclear power plants is presented.

\section{Wireless LAN standards}

The Institute of Electrical and Electronics Engineers (IEEE) has been produced a series of standards for wireless networks referred as 802.11x, for wireless LAN (Local Area Networks). The original standard used to implement wireless LANs was 802.11 (IEEE, 1999a). It was first published in 1999 and designed to support a maximum data rate of 2 Mbps in the $2.4 \mathrm{GHz}$ band. This standard uses two modulation techniques: frequency hopping spread spectrum (FHSS) and direct sequence spread spectrum (DSSS). Figure 2 shows the IEEE 802.11 standard architecture.

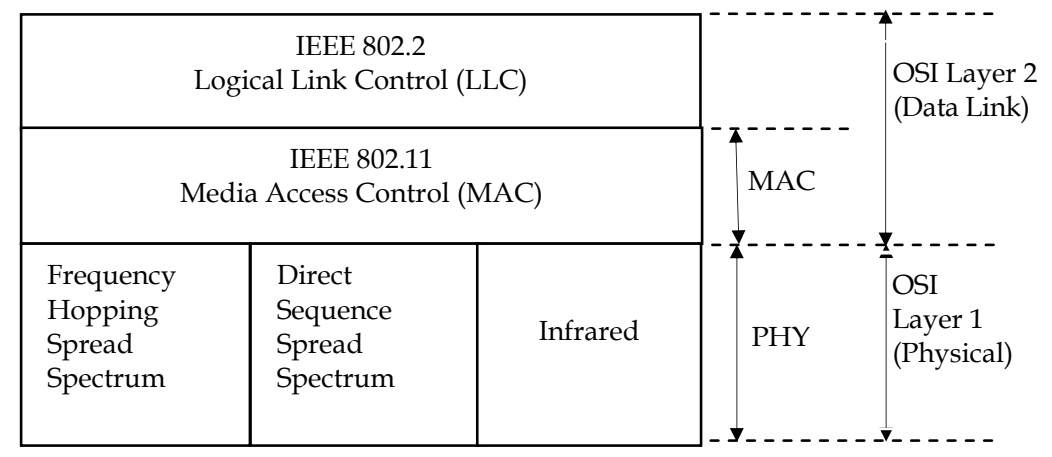

Fig. 2. The IEEE 802.11 Standard Architecture

\subsection{The IEEE 802.11b standard}

Wireless Fidelity (Wi-Fi) networks are intended to be implemented more in enterprises and in industry. Wi-Fi is commonly used as the abbreviation of $802.11 \mathrm{~b}$ standard. It supports 
bandwidth up to $11 \mathrm{Mbps}$, comparable to traditional Ethernet. The $802.11 \mathrm{~b}$ standard also uses DSSS as modulation technique in the $2.4 \mathrm{GHz}$ band as the original 802.11 standard, where Wi-Fi devices communicate to each other at data rates up to $11 \mathrm{Mbps}$ (IEEE, 1999b). In case of any radiofrequency or interference signal cause errors in data transmission, Wi-Fi devices will automatically reduce their data rate to $5.5 \mathrm{Mbps}$, to $2 \mathrm{Mbps}$ and finally to 1 Mbps (Martínez, 2002). These occasional reductions in the data rate are aimed to keep the network very stable and highly reliable. Some advantages of Wi-Fi standard are: high data rates, reliable, wide signal ranges and supports of all 802.11 devices using DSSS.

\subsection{Other IEEE 802.11x standards}

Along with the 802.11, other standards have been developed (Martínez, 2002): 802.11a, 802.11g, 802.11e and 802.11i. The 802.11a standard (IEEE, 1999c) was published in 1999 and uses Orthogonal Frequency Division Multiplexing (OFDM) to support bandwidth up to 54 Mbps and signals in the unlicensed frequency spectrum around $5.8 \mathrm{GHz}$. This higher frequency compared to $802.11 \mathrm{~b}$ limits the range of $802.11 \mathrm{a}$ networks. The higher frequency also means 802.11a signals have more difficulty penetrating walls and other obstructions. To solve this problem and to cover a similar range that $802.11 \mathrm{~b}$, a greater number of access points must be installed. Because $802.11 \mathrm{a}$ and $802.11 \mathrm{~b}$ utilize different frequencies, the two technologies are incompatible with each other. The 802.11g standard (IEEE, 2003) attempts to combine the best of both $802.11 \mathrm{a}$ and $802.11 \mathrm{~b}$ standards. It uses OFDM to support bandwidth up to $54 \mathrm{Mbps}$, using the $2.4 \mathrm{GHz}$ band for greater range. $802.11 \mathrm{~g}$ is backwards compatible with $802.11 \mathrm{~b}$, meaning that $802.11 \mathrm{~g}$ access points will work with $802.11 \mathrm{~b}$ wireless network adapters and vice versa. Also, $802.11 \mathrm{~g}$ produces less multipath losses than 802.11a. The 802.11e standard (IEEE, 2005) has been developed to support applications with quality of service (QoS) requirements. It supports a wide range of real-time applications such as voice, audio, video and data over WLAN. It adopts time scheduling and polling mechanisms to cope with delay sensitive traffic. The 802.11i standard (IEEE, 2004) has been developed to improve the security of data provided by Wired Equivalent Privacy (WEP) protocol in 802.11b. 802.11i incorporates an entirely new privacy algorithm and authentication mechanism based on the Advanced Encryption Standard (AES).

\section{Electromagnetic interference standards}

Electromagnetic interference (EMI), radio-frequency interference (RFI), and power surges have been identified in (NUREG, 2003) as environmental conditions that can affect the performance of safety-related electrical equipment in a nuclear power plant. A series of comprehensive commercial EMI/RFI immunity standards have been issued by the following international organizations:

- International Electrotechnical Commission (IEC)

- European Committee for Electrotechnical Standardization (CENELEC)

- International Special Committee on Radio Interference (CISPR)

These international organizations have produced standards for general application. National organizations in countries like United States of America, Canada, Australia and Europe have their own standards that regulate EMI/RFI immunity of electronic equipment. In the U.S., the Nuclear Regulatory Commission (NRC) has produced the most comprehensive guide known as Regulatory Guide 1.180 (NRC, 2003) with the aim of 
developing the technical basis for regulatory guidance to address electromagnetic interference, radio-frequency interference, and surge withstand capability in safety-related instrumentation and control systems in a nuclear power plant.

\subsection{Regulatory guide $\mathbf{1 . 1 8 0}$}

This regulatory guide endorses design, installation, and testing practices acceptable to the NRC staff for addressing the effects of EMI/RFI and power surges on safety-related instrumentation and control systems in a nuclear power plant environment. This regulatory guide is based on the standards: IEEE 10-50 (IEEE, 1996), MIL-STD-461E (DoD, 1999) and IEC 61000 series (IEC, 2002). For instance, the design and installation practices described in the IEEE 1050 standard are endorsed for limiting EMI/RFI subject to the conditions stated in the Regulatory Position. Electromagnetic compatibility (EMC) testing practices from military and commercial standards are endorsed to address electromagnetic emissions, EMI/RFI susceptibility, and power surge withstand capability. The MIL-STD-461E standard contains test practices that can be applied to characterize EMI/RFI emissions. In addition, selected EMI/RFI test methods from MIL-STD-461E and the IEC 61000 series are endorsed to evaluate conducted and radiated EMI/RFI phenomena for safety-related control and instrumentation systems. The IEC electromagnetic compatibility (EMC) standards include IEC 61000-3 (part 3: Limits), IEC 61000-4 (part 4: Testing and measurement techniques), and IEC 61000-6 (part 6: Generic standards). This regulatory guide also endorses electromagnetic operating envelopes corresponding to the MIL-STD-461E test methods. These operating envelopes were tailored from the MIL-STD-461E test limits to represent the characteristic electromagnetic environment in key locations at nuclear power plants. Comparable operating envelopes for the IEC 61000 test methods are also endorsed. The Regulatory Guide 1.180 was updated in the year 2003 to provide additional acceptable methods for validating the performance of instrumentation and control systems and includes guidance on testing to address signal line susceptibility and very high frequency $(>1 \mathrm{GHz})$ phenomena.

\section{Wireless LAN technologies}

The use of wireless technologies in industrial and power utility environments, including their use in nuclear power plants is growing fast. The wireless technologies that have been implemented in nuclear power plants include paging systems, digital mobile radio and cellular systems. Currently, WLAN technology is been installing and evaluating in nuclear power plants, due to it provides enhanced features compared to traditional wireless communications technologies such as conventional mobile radio in two key aspects:

- Higher operation frequencies

- Lower output power

WLAN technology is based on the 802.11 standard and generally operates at higher frequencies $(2.4 / 5 \mathrm{GHz})$ and at significantly lower effective power output levels $(20 \mathrm{~mW}$ 1W) than UHF/VHF $(890 / 450 \mathrm{MHz})$ communication systems operating at power output levels between $4-5 \mathrm{~W}$. This feature of more modem wireless devices, including WLANs, generally requires that the end user be closer to a potentially sensitive device before interference is noted. So, it can be inferred that modem wireless devices are less of a threat and less like1y to interfere with nuclear power plant equipment than older devices; that is, 
more modern devices tend to be less intrusive. Those features make that the electromagnetic interference generated by devices based on WLAN technology does not affect significantly to safety-related instrumentation and control equipment (EPRI, 2003). In 2002, the Electric Research Institute (EPRI) published a report in which EPRI developed guidelines for the use of wireless technologies in nuclear power plants (EPRI, 2002). The purpose of this report was the evaluation of wireless technologies in nuclear power plants for integrated (voice, data and video) communication, remote equipment and system monitoring, and to complement an electronic procedures support system. The guidelines effort focuses on the development of a rules structure to support the deployment of wireless devices in a nuclear power plant without compromising continuous, safe, and reliable operation. For these reasons, spread spectrum appears to be the most adequate technology for the nuclear power environment.

\subsection{Frequency spectrum regulation}

The Federal Communications Commission (FCC), organism that manages and regulates the electromagnetic spectrum in U.S. assigned in 1985 to ISM band the $900 \mathrm{MHz}, 2.4 \mathrm{GHz}$ and $5.8 \mathrm{GHz}$ frequency ranges. These regulations are specified in the CFR-47 section 15.2.47 (FCC, 2004). The ISM band is a license-free band and it is used by WLAN technology. In Mexico, the Consultative Committee for Standardization in Telecommunications (CCNNT) manages and regulates the electromagnetic spectrum (CCNNT, 2001). Table 1, shows the frequency ranges and bandwidth reserved for the ISM band for their use the U.S. and Mexico.

\begin{tabular}{|c|c|}
\hline ISM Band & Bandwidth \\
\hline $902-928 \mathrm{MHz}$ & $26 \mathrm{MHz}$ \\
\hline $2.4-2.4835 \mathrm{GHz}$ & $83.5 \mathrm{MHz}$ \\
\hline $5.725-5.850 \mathrm{GHz}$ & $125 \mathrm{MHz}$ \\
\hline
\end{tabular}

Table 1. Reserved frequencies for the ISM band

The FCC set these ISM bands for license-free and low power radio transmission over short to medium distances (Meel, 1999). The FCC requires that the signal be distributed over a wide swath of bandwidth using a spread spectrum technology originally developed by the military for anti-jamming applications. Wireless devices that operate in these license-free bands can allow immediate, real-time commissioning of a network, avoiding the delays associated with installing wiring or cables. By spreading data transmissions across the available frequency band in a prearranged scheme, spread spectrum encoding technology makes the signal less vulnerable to noise, interference, and snooping. The significant amount of metal often found in industrial settings can cause signals sent over a single frequency to bounce and cancel other signals arriving at the same time. Spread spectrum technology helps overcome this problem and allows multiple users to share a frequency band with minimal interference from other users.

\subsection{Radiated power regulation}

The power level radiated by an antenna in a WLAN is specified by the rules stated in the FCC section 15.247 for operation in the U.S. (FCC, 2004). The FCC also limits the increase in 
the output power of the antenna to a maximum of $6 \mathrm{dBi}$. So, the radiated power is limited to a maximum of $1 \mathrm{~W}$ for the $2.4 \mathrm{GHz}$ band. In Mexico it is limited to a maximum of $650 \mathrm{~mW}$ (CCNNT, 2001). In Europe and Japan, the radiated power levels are different to those allowed for U.S. and Mexico. Table 2, shows the maximum radiated power levels allowed for different countries (Meel, 1999).

\begin{tabular}{|c|c|c|}
\hline $\begin{array}{c}\text { Maximum } \\
\text { Transmit Power }\end{array}$ & $\begin{array}{c}\text { Geographical } \\
\text { Location }\end{array}$ & Compliance Document \\
\hline $1000 \mathrm{~mW}$ & U.S.A. & FCC 15.247 \\
\hline $650 \mathrm{~mW}$ & Mexico & CCNN-T \\
\hline $100 \mathrm{~mW}$ & Europe & ETS 300-328 \\
\hline $10 \mathrm{~mW} / \mathrm{MHz}$ & Japan & $\begin{array}{c}\text { MPT ordinance for } \\
\text { Regulating Radio } \\
\text { Equipment, article 49-20 }\end{array}$ \\
\hline
\end{tabular}

Table 2. Maximum transmit power

\subsection{Bandwidth regulation}

Data throughput is adversely affected by distance and the amount of noise or interference in the area. If too many wireless devices are operating in the same vicinity, they can interfere with each other, restricting network capacity. In terms of protection from interference, the FCC and CCNN-T specify that WLANs operating in the three ISM bands, use spread spectrum (SS) as the encoding technique to comply with regulation requirements (Meel, 1999; DoE, 2002; Pearce, 2001). Spread spectrum technology is based on two interference avoidance techniques: frequency hopping spread spectrum (FHSS) and direct sequence spread spectrum (DSSS). Both modulation schemes have been defined in (IEEE, 1999b) to operate in the $2.4 \mathrm{GHz}$ band, using a bandwidth of $83 \mathrm{MHz}$ (from $2.400 \mathrm{GHz}$ to $2.4835 \mathrm{GHz}$ ). Also, the CCNNT specifies that the bandwidth of the transmitted signal depending on the modulation scheme (FHSSS or DSSS) employed (CCNNT, 2001), as shown in table 3. FHSS technique permits the fast movement or "hopping" to any channel within the total allocated spectrum. Here, the carrier frequency hops from channel to channel in some pre-arranged sequence. The major drawback to this technique is a limited data rate.

\begin{tabular}{|c|c|}
\hline $\begin{array}{c}\text { SS Modulation } \\
\text { scheme }\end{array}$ & Bandwidth \\
\hline $\begin{array}{c}\text { Frequency Hopping } \\
\text { (FHSS) }\end{array}$ & $1 \mathrm{MHz}$ (maximum) \\
\hline Direct Sequence (DSSS) & $\begin{array}{c}500 \mathrm{KHz} \\
\text { (minimum) }\end{array}$ \\
\hline
\end{tabular}

Table 3. Bandwidth of transmitted signal

By contrast, DSSS technique provides much higher data rates. Here, the carrier frequency does not jump from frequency to frequency, but instead spreads the information across a much wider bandwidth. Also, DSSS can provide many users to be on the same channel at the same time and be distinguished from each other by a digital code. DSSS uses the phase shift keying modulation technique known as differential BPSK (DBPSK) and differential BPSK (DQPSK). FHSS uses the frequency shift keying modulation technique known as 2 
and 4 level Gaussian FSK (2GFSK and 4GFSK). The data rate for both FHSS and DSSS schemes is defined in (IEEE, 1999a; IEEE, 1999b). Table 4, shows the maximum data rates for the physical layer supported by the most commonly used 802.11 standards. The most common problem when applying an unlicensed wireless system in an industrial environment is radio frequency interference or better known as "radio noise". Both FHSS and DSSS handle noise differently and can have certain advantages depending on the type of interference experienced.

\begin{tabular}{|c|c|c|c|}
\hline Standard & $\begin{array}{c}\text { Frequency } \\
\text { band (GHz) }\end{array}$ & $\begin{array}{c}\text { Modulation } \\
\text { scheme }\end{array}$ & $\begin{array}{c}\text { Maximum bit } \\
\text { rate }\end{array}$ \\
\hline IEEE 802.11 & $2.4-2.4835$ & FHSS & $2 \mathrm{Mbps}$ \\
\hline IEEE 802.11 & $2.4-2.4835$ & DSSS & $2 \mathrm{Mbps}$ \\
\hline IEEE 802.11b & $2.4-2.4835$ & DSSS & $11 \mathrm{Mbps}$ \\
\hline
\end{tabular}

Table 4. Maximum data rates for WLANs

\section{Use of wireless LANs in the nuclear environment}

The WLAN technology based on the IEEE 802.11 standard has a very promising future for its use in nuclear power plants due to features like mobility, reliability, security, scalability and compatibility with other communication networks technologies in order to provide new services such as voice over IP (VoIP) and IP video (Shankar, 2003). A key issue of these technologies is that no wires are needed to implement new services. Currently, WLAN technology is been installing and evaluating in nuclear power plants, due to it provides enhanced features compared to traditional wireless communications technologies such as conventional mobile radio in two key aspects: higher operation frequencies and lower output power which translates in very high data rates and very low electromagnetic interference. However, wireless technology may exhibit greater vulnerability to the nuclear power plant EMI/RFI environment than existing instrumentation and control systems. The typical environment in a nuclear power plant includes many sources of electromagnetic interference (EMI), radio-frequency interference (RFI), and power surges, such as hand-held two-way radios, arc welders, switching of large inductive loads, high fault currents, and high-energy fast transients associated with switching at the generator or transmission voltage levels. Hence, operational and functional issues related to safety in the nuclear power plant environment are required to address the possibility of troubles and malfunctions in instrumentation and control systems caused by EMI/RFI and power surges.

The wireless communications technologies that can be implemented in nuclear power plants include paging systems, mobile radio, cellular systems and wireless local area networks (WLANs). Recently, there has been a significant increase in the use of wireless technology in nuclear power plants. For example, wireless technology has been installed and evaluated in nuclear power plants by utility companies throughout the U.S., Canada, Mexico, Europe, and worldwide (Bahavnani, 2001; Telrad Connegy, 2001; Wireless Magazine, 1995). Wireless technology has many applications in the industry, including cellular phone systems, paging systems, two-way radio communication systems, dose management in nuclear power plants, remote monitoring and tracking systems, and operator logs (EPRI, 2004a). In addition, EPRI has reported the following case studies: Exelon's Peach Bottom Nuclear 
Power Plant, Texas Utilities Comanche Peak Nuclear Station, and in the San Onofre Nuclear Generating Station de la Southern California Edison (EPRI, 2004b). Other application cases of wireless technology in nuclear power plants in the U.S. include: Nine Mile Point Nuclear Station, Robinson Nuclear Plant (CP\&L) and in the Seabrook Nuclear Power Plant (Shankar, 2003; SpectraLink, 2004; Kjesbu, S. and Brunsvik T., 2000).

\section{Mobile IP telephony system for a nuclear power plant}

Mobile IP telephony is the new generation of communications networks that makes possible the convergence of voice and data over wireless local area networks. It refers to the transmission and reception of voice conversations over wireless IP data networks. This technology combines data networks with mobile technologies to support voice and data applications over a common integrated network. A key advantage of IP telephony is that it allows the transmission of voice signals from wireless telephones over IEEE 802.11 wireless LANs connected to an IP network backbone, being either a public network (Internet) or a private network (Intranet). IP telephony is designed to replace the legacy TDM (time division multiplexing) technologies and networks with an IP-based data network. Digitized voice will be carried in IP data packets over a LAN and/or WAN network. A major aspect involved in a voice conversation using mobile IP telephony is the conversion of analog or digital voice signals from conventional phones to IP packets for further transmission either to a fixed or mobile phone, over an IP network.

This section presents the design considerations for the implementation of a mobile IP telephony system for voice communications applications in Laguna Verde nuclear power plant (CNLV), Federal Commission of Electricity (CFE), Mexico based on national and international standards as well as, the technical requirements that commercially available equipment must meet. In addition, the technical requirements of a mobile IP management system are presented.

\subsection{Components of the mobile IP telephony system}

One of the main requirements of a mobile IP telephony system is that the proposed system must meet the design technical requirements for its exclusive operation in a nuclear power plant in Mexico. Regarding standardization, it must meet national and international standards and regulatory guides applicable to nuclear power plants. Also, this system will operate upon the existing CNLV's data backbone which is based on Gigabit Ethernet switching technology. The model proposed in this work for the mobile IP telephony system is showed in figure 3 and it is composed of the following components:

- Voice gateway

- Priority server

- Wireless Access points (AP)

- Wireless telephones

The voice gateway provides the conversion of analog or digital voice signals from conventional phones to IP packets for further transmission to any access point connected to the CNLV's IP data network backbone. The priority server has as a main function to assign priorities according to the type of traffic to be sent over the network, in order to give the highest priority to voice packets guaranteeing in this way high quality voice conversations. The wireless access points have as a main function to interconnect the portable wireless 
mobile telephones to the CNLV's backbone. The functionality of the proposed system shall be so good that allows any user located in a wireless coverage area using a conventional analog or digital telephone connected to a private branch exchange (PBX) equipment to make calls and communicate with any other user located in some other area carrying a wireless telephone assigned to an access point or having nearby a conventional wired telephone.

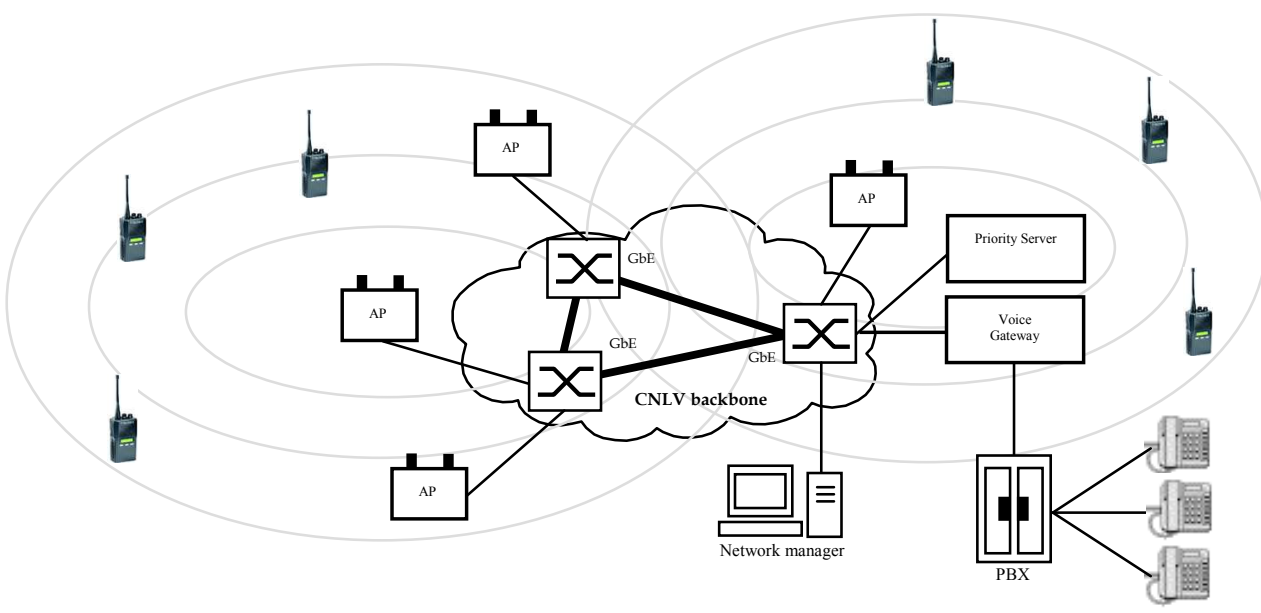

Fig. 3. Mobile IP telephony system for the CNLV nuclear plant, Mexico.

In addition, a network management system for the mobile IP telephony system is proposed. It is composed of a server and the network management software which will allow visualizing all network components such as access points, wireless telephones, voice gateways, priority server and airspace by means of a human machine interface (HMI). Next, the technical requirements that every component of the proposed mobile IP telephony system shall meet, are presented.

\subsection{Technical requirements of the mobile IP telephony system}

The proposed mobile IP telephony system shall support voice over IP communication applications within the CNLV controlled areas. Regarding standardization, it must meet national and international standards and regulatory guides applicable to nuclear power plants. The components of the wireless system shall be compatible with IEEE $802.11 \mathrm{~b}$ standard (IEEE, 1999b), use direct sequence spread spectrum (DSS) a modulation technique and support a data rate of $11 \mathrm{Mbps}$. The systems must operate in the ISM (Industrial, Scientific and Medical) band from 2.4 to $2.4835 \mathrm{GHz}$, according to the NOM-121-SCT-94 standard (CCNNT, 2001) from the Mexican Normalization in Telecommunications Consultative Committee. The maximum output power radiated form the wireless components shall be $100 \mathrm{~mW}$. The system shall be composed of industrial type equipment for use in nuclear power plants and must meet the acceptable electromagnetic interference (EMI) and radiofrequency interference (RFI) ranges for electronic equipment operating in frequencies above of I GHz, established in the regulatory guide 1.180 (NRC, 2003), issued by the United States Nuclear Regulatory Commission (NRC). The system must meet military 
levels of electromagnetic emissions, electromagnetic compatibility and electromagnetic interference susceptibility for electronic equipment operating in the frequency range from 1 GHz to $10 \mathrm{GHz}$, according to MIL-STD-461E (DoD, 1999) military standard. Also, the system must meet the applicable electromagnetic interference and radiofrequency interference standards from the International Special Committee on Radio Interference (CISPR), the International Electrotechnical Commission (IEC) as well as, from the Institute of Electrical and Electronics Engineers (IEEE).

The system must meet the technical requirements of electromagnetic compatibility (EMC) and radiofrequency interference contained in the IEC-61000 (IEC, 2002) set of standards. It must meet national and international wireless communication standards such as NOM-121SCT-94 (CCNNT, 2001), FCC Part 15.247 (FCC, 2004), IEEE 802.3 (IEEE, 2002) and IEEE 802.11b (IEEE, 1999b). Regarding the system components, they must be designed to operate in risky and severe environments such as: high humidity levels, temperature, radiation, noise, vibration, shock, as well as in controlled levels in a nuclear power plant. Regarding security, the system shall provide military like high security levels by using data encryption techniques as integral part of the voice over IP communication system. Also, the system must use robust electronic equipment and provide an availability at least of $99.999 \%$, considering a redundant and fault tolerant communications architecture, as well as an uninterruptible power supply (UPS) with the aim of avoiding the system collapse.

The system shall be designed with the necessary security and authentication levels in order to deny the access to private information of validated users or their privileges by means of interception and modifications of their data messages. In addition, it must provide high confidentiality in order to guarantee very high efficiency in the activation and deactivation of voice conversations, data messages, control messages or authentication passwords. With regard to availability, then mobile IP telephony system must guarantee the continuous voice communications between mobile users using wireless telephones and the access points installed in and out the CNLV power plant. Regarding wireless telephones, the system must handle 64 spread spectrum wireless telephones per unit, being 128 in total to serve the 2 units available to the CNLV.

\subsubsection{Voice bandwidth}

In the traditional voice world a single T1 leased line is used to carry 24 digital channels of 64 Kbps, toll quality telephone calls from the public switched telephone network (PSTN). In its turn, those with a private point-to-point T1 connection can compress the voice to less than $64 \mathrm{Kbps}$ bandwidth for more efficiency, but the quality may be sacrificed. The three most common modulation schemes for encoding voice are:

- $64 \mathrm{Kbps}(\mathrm{PCM}) / 1.544 \mathrm{Mbps}=24$ simultaneous calls on a T1

- $\quad 32 \mathrm{Kbps}(\mathrm{ADPCM}) / 1.544 \mathrm{Mbps}=48$ simultaneous calls on a T1

- $8 \mathrm{Kbps}(\mathrm{CELP}) / 1.544 \mathrm{Mbps}=120$ simultaneous calls on a T1

Bandwidth use and voice compression play an important role in provisioning the LAN/WAN.

\subsubsection{Voice over IP bandwidth}

The concept of combining traditional voice on data networks is simple because voice traffic uses a lot less bandwidth than traditional LAN-based computer networks. A single tollquality phone call over the public network uses $64 \mathrm{Kbps}$ in each direction - that's only 
$0.0625 \%$ of a 100 Mbps full duplex link. On a 100 Mbps Ethernet network, each voice call takes up to $85.6 \mathrm{Kbps}$ (64 Kbps + IP header + Ethernet header) in each direction supporting up to 1,160 calls over a full duplex link. On a Gigabit backbone, up to 11,600 simultaneous calls can be handled.

\subsubsection{Voice gateway}

The voice gateway has as main function to provide the conversion of analog or digital voice signals from conventional phones to IP packets for further transmission to any access point connected to the CNLV's IP data network backbone. For the implementation of the mobile IP telephony system, it is required to integrate the analog or digital voice circuits from the private branch exchange (PBX) Harris equipment model 20-20L installed in the CNLV to a voice over IP system based on an Ethernet wireless LAN, and compatible with the IEEE 802.11b standard (IEEE, 1999b). This function must be carried out by the proposed voice gateway. Next, the most relevant technical requirements the voice gateway shall meet are presented.

Regarding the functional characteristics, the proposed voice gateway must provide the interface between an enterprise PBX and a wireless LAN network by supporting digital and/or analog line interfaces, and shall provide the voice encoding and packetization required to transmit telephone conversations over an IP network. It must provide RJ-21 physical interfaces for its connection to the PBX, as well as a RJ-45 interface for connection to an Ethernet LAN. It must provide the necessary control signaling to the host PBX allowing wireless telephone users to access all the features and capabilities of the PBX. The setting up of voice connections and the control of voice calls within the voice gateway shall support a wide variety of packet-oriented protocol standards such as ITU-H.323 (ITU, 2009), RFC3261 (IETF, 2002), ITU-H.248.1 (ITU, 2005) or a subset of these protocols. Also, the voice gateway shall support H.323-compliant clients, allowing third-party wireless devices to make and receive calls through the host PBX. In addition, the voice gateway must provide coding and decoding of voice packets according to VoIP compression standards such as ITU-G.711 (ITU, 1988), ITU-G723.1 (ITU, 2006)or ITUG.729 A/B (ITU, 2007) with design characteristics that enhance the quality of the voice signals to be transmitted providing silence suppression and echo cancellation functions according to the ITU-G.168 standard (ITU, 2009).

With regard to security and availability, the voice gateway to be used in a nuclear power environment shall meet military requirements of electromagnetic interference emissions, compatibility and susceptibility specified in MIL-STD-461E (DoD, 1999) and IEC-61000 (IEC, 2002) standards. In its turn, it must meet security technical requirements applicable to electronic communication equipment stated in the IEC-60950 (IEC, 2005) standard or other equivalent standards like UL-60950 y EN-60950.

\subsubsection{Priority server}

The proposed priority server has as a main function to recognize and to assign priorities to voice and data packets according to the type of traffic to be sent over the network, in order to give the highest priority to voice packets to reduce the voice packets time delay and causing the minimum impact on data throughput, guaranteeing in this way high quality voice conversations. Next, the most relevant technical requirements the priority server shall meet are presented. 
The proposed priority server shall use a packet filtering mechanism to guarantee a high quality of service (QoS) to voice packets with the possibility of a transparent migration of the system to the IEEE 802.11e standard (IEEE, 2005) when it has been ratified. It must has the capacity of supporting up to 120 simultaneous voice calls, provide an Ethernet 100Base$\mathrm{T}$ port with a RJ-45 connector, support full-duplex transmission compatible with the IEEE 802.11b standard (IEEE, 1999b) for operation in Wi-Fi WLANs and also, it must be capable of supporting native VoIP protocols and interfaces provided by the proposed voice gateway. With regard to the WLAN monitoring, the priority server shall provide a RS-232 interface for configuration and diagnostics purposes, WLAN connection indicators, system activity, collisions events and error state indicators. It shall support local mode configuration by using the RS-232 and USB interfaces or support remote mode configuration by using the Telnet application protocol over a LAN or WLAN.

Regarding the operational conditions, the priority server shall support an operation temperature range from $0^{\circ} \mathrm{C}$ to $40^{\circ} \mathrm{C}$, relative humidity between $0 \%$ and $95 \%$ noncondensed, and a supply voltage of 110/220 VAC at an operation frequency of $60 \mathrm{~Hz}$.

\subsubsection{Wireless access points}

The proposed wireless access points have as a main function to interconnect the portable wireless mobile telephones to the CNLV nuclear power plant's data backbone. According to the normative defined by the Mexican Normalization in Telecommunications Consultative Committee (CCNNT, 2001) as well as, for the CNLV nuclear power plant environment operating conditions, the access points providing the wireless communication links to the mobile telephones must operate in the ISM frequency band, from 2.4 to $2.4835 \mathrm{GHz}$ an must use a maximum output radiated power of $100 \mathrm{~mW}$. Next, the most relevant technical requirements the wireless access points shall meet, are presented.

For the implementation of the wireless VoIP application, the proposed wireless access points shall are required to be compatible with the IEEE 802.11b (IEEE, 1999b) standard, use direct sequence spread spectrum modulation technique and support a data rate of $11 \mathrm{Mbps}$. With regard to security, the wireless access points shall provide very high security mechanisms to voice and data packets during transmissions of voice conversations by supporting at least the WEP (Wired Equivalent Privacy) encryption technique with 128 bit keys, and the possibility of easily migrate to the WPA (Wi-Fi Protected Access) encryption scheme, as well as to support the security mechanisms included in the IEEE 802.11i standard (IEEE, 2004).

Other security functions are desirable such as continuous airspace monitoring, detection and blocking of access points that are not part of the CNLV's data backbone, etc. The wireless access points shall be compatible with quality of service mechanism supported by the proposed priority server and the voice gateway, with the possibility of migrating in a transparent manner to the IEEE 802.11e (IEEE, 2005) when it has been ratified. The physical distribution and the number of the wireless access points installed in the CNLV nuclear power plant must guarantee a wireless coverage area of $100 \%$ in each controlled area so the use of dual external antennas shall allow a much better distribution of electromagnetic signals radiation pattern so that no black spots exist.

In order to improve the reliability and availability of the mobile IP telephony system, the wireless access points shall provide two 10/100 Mbps Ethernet ports one for physical connection and the other one for a backup network segment. The power supply for the wireless access points shall be provided by means of a UTP network cable supporting Power 
over Ethernet (PoE) mechanism, according to the IEEE 802.3af standard (IEEE, 2003). In addition, it is recommended that each wireless access point shall provide an independent $110 / 220$ VAC voltage input.

The legislation that the wireless access points must meet, includes the regulation emitted by the Federal Communications Commission, FCC Part 15.247 (FCC, 2004) for digitally modulated intentional radiators devices, and the security and electromagnetic interference requirements (DoD, 1999), (IEC, 2002), (IEC, 2005), in order to respect the acceptable electromagnetic interference and radiofrequency ranges for electronic communication equipment operating at frequencies above $1 \mathrm{GHz}$ according to the Nuclear Regulatory Guide 1.180 (NRC, 2003), emitted by the Nuclear Regulatory Commission.

\subsubsection{Wireless telephones}

The proposed wireless telephones will be used by personnel working in the external areas of the CNLV nuclear power plant, conducting fieldwork so that they have to be robust designed for using in industrial and nuclear power plants, in particular. Next, the most relevant technical requirements the wireless telephones shall meet, are presented.

The wireless telephones shall be compliant to the IEEE 802.11b (IEEE, 1999b), H.323 (ITU, 2009), G.711 (ITU, 1988), G.729 (ITU, 2007) standards as well as to VoIP protocols emitted by international standards bodies. Besides, they must support the capability of sending and receiving short text messages via open application interface. The wireless telephones shall support both static and dynamic (DHCP) IP addressing configuration and must operate in the ISM frequency band, from 2.4 to $2.4835 \mathrm{GHz}$, according to the NOM-121-SCT-94 standard (CCNNT, 2001), issued by the Mexican Normalization in Telecommunications Consultative Committee. They shall be compliant to the IEEE 802.11b (Wi-Fi) standard (IEEE, 1999b), use direct sequence spread spectrum (DSSS) modulation technique and support data rates of 11,5.5, 2 and $1 \mathrm{Mbps}$, which must be automatically selected according to the communication channel conditions and voice quality of service.

With regard to radiated power, the wireless telephones shall produce a maximum transmission power below $100 \mathrm{~mW}(20 \mathrm{dBm})$, which must be automatically adjusted in order to have always the same radiated power level. They shall provide very high security mechanisms to voice and data packets during transmissions of voice conversations by supporting at least the WEP (Wired Equivalent Privacy) encryption technique with 128 bit keys, and the possibility of easily migrate to the WPA (Wi-Fi Protected Access) encryption scheme, as well as to support the security mechanisms included in the IEEE 802.11i standard (IEEE, 2004).

In addition, the proposed wireless telephones shall provide an LCD backlit dot matrix display with icons and line-status indicators with the aim of visualizing the entire display in darkness conditions. They shall support the instant communication feature known as pushto-talk (PTT) by using IP multicast addresses. This requires that multicasting be enabled on the subnet used for the wireless telephones, priority server, and voice gateway. They shall provide an integrated TFTP client in order to allow remote software updates via the TFTP (Trivial File Transfer Protocol) application. Also, wireless telephones must be lightweight with a weight less than 200 grams.

\subsection{Mobile IP management system}

In addition to the mobile IP telephony system, a network management system is proposed. It consists of the network management server and the network management software. Next, 
the most relevant technical requirements the network management system shall meet, are presented.

The proposed network management server shall provide the following minimum capacities: $1.8 \mathrm{GHz}$ processor (Pentium IV), $256 \mathrm{MB}$ RDRAM, internal $40 \mathrm{~GB}$ hard disk, a CD-ROM unit, a 20" color monitor, and a 10/100 Mbps Ethernet network card. For its part, the network management software must be capable of visualizing all components of the mobile IP telephony system such as access points, wireless telephones, voice gateway, and priority server) as well as the airspace. With regard to capacity, the network management software shall provide management functions like configuration, performance monitoring, fault detection, network statistics, and security, among others. Regarding functionality, it shall support functions such as discovering, configuring and monitoring all access points connected to de CNLV data backbone, allowing the configuration all wireless devices specified in the design of the mobile IP telephony system with just one click.

In addition, the network management system shall provide management tools such as monitoring and measurement of the wireless network performance (delay, throughput, etc.), used and available bandwidth, wireless network use, among others parameters. It shall provide wireless network statistics such as transmitted and received signal level, number of transmitted and received IP packets, frequency deviations, and changes in data rate for each access point.

With regard to security, the network management system must be a centralized-type system, and be capable of providing the mobile IP telephony system with a high level of security by means of monitoring both the physical network devices and the wireless pace used by the system. Also, it shall detect most of wireless network cyber attacks including massive attacks, intrusions, impersonation, sniffers, denial of service (DoS), etc., and finally the network management system must has the ability to perform remote software upgrades to wireless telephones from the network management's central station.

\subsection{Implementation of the mobile IP telephony system at CNLV}

In this section, an example of use of the proposed mobile IP telephony system for voice communications applications in Laguna Verde nuclear power plant (CNLV) is presented. Once the design considerations for the implementation of a mobile IP telephony for voice communications applications were carried out, the Federal Commission of Electricity (CFE), Mexico began the system acquisition phase with an international bidding in order to have a winner. Then, the components of the mobile IP telephony system such as: access points, voice gateway, priority server, and wireless telephone, etc., were supplied and installed in the selected controlled areas of the CNLV nuclear power plant. After this, the implementation phase began. The acquired mobile IP telephony system was installed at CNLV's telecommunications room, and now it is operating upon the existing CNLV's data backbone which is based on Gigabit Ethernet switching technology. The system provides communication applications such as telephony and voice over IP.

Another example of use of wireless LAN technologies in the nuclear power plant environment from the previous project is that, CFE has initiated a new implementation phase consisting of the introduction of wireless IP video technology with the aim of having a true integrated data, voice and video system using the same CNLV's network infrastructure. The proposed IP video system will be used for remote video monitoring and video surveillance within the CNLV nuclear power plant taking advantage of the IEEE $802.11 \mathrm{~b} / \mathrm{g}$ standard-based wireless 
network technology already installed. The main components of the system are: wireless IP video cameras, massive storage unit (terabyte network attached storage), and a video monitoring and surveillance station. The proposed IP video system which will be integrated to the existing wireless network is shown in figure 4.

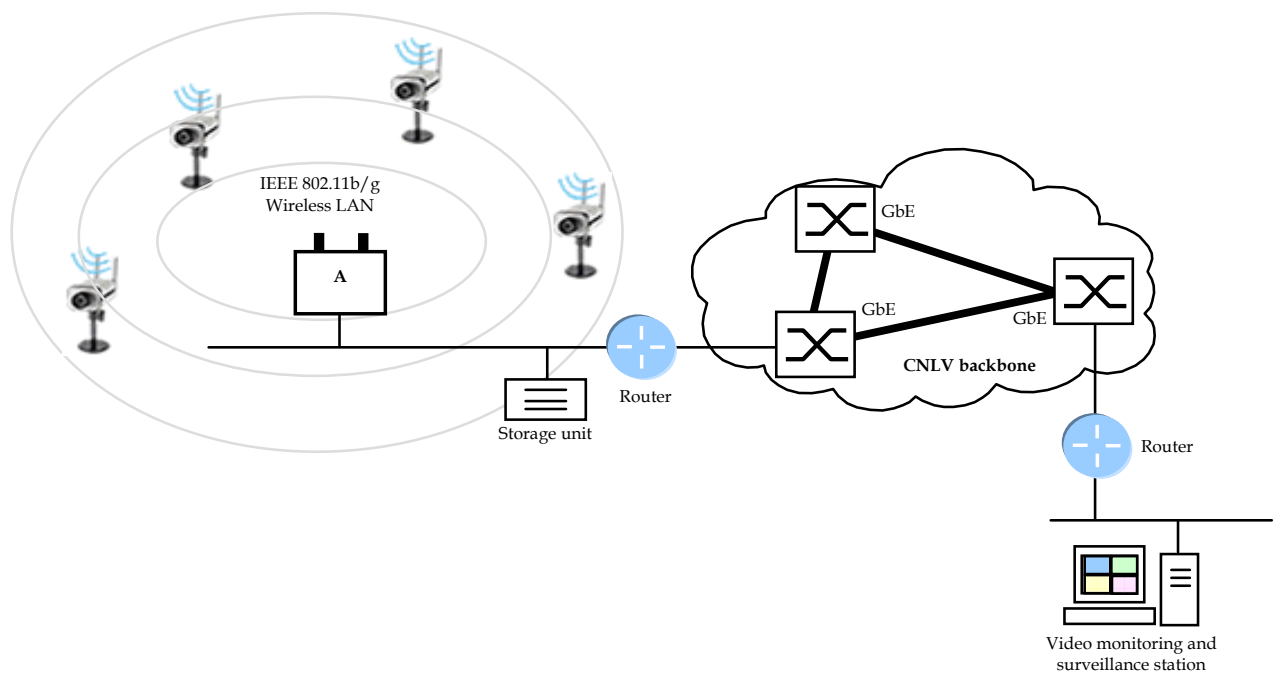

Fig. 4. Proposed wireless IP video system for the CNLV nuclear plant, Mexico.

\section{Conclusions}

In this chapter, the design considerations for the implementation of a mobile IP telephony system for voice communications applications in Laguna Verde nuclear power plant (CNLV), Federal Commission of Electricity (CFE), Mexico based on national and international standards were presented. Also, this work gave an analysis of the most relevant wireless technologies currently available that can be implemented in nuclear power plants and also identified nuclear regulatory guidelines, wireless networks standards, electromagnetic and radio-frequency interference standards. With regard to the use of wireless LANs in the nuclear environment, there is clear evidence that the electromagnetic interference and radio-frequency interference conditions can adversely affect the performance of safety-related instrumentation and control equipment. EMC is an element of addressing that requirement. Operational and functional issues related to safety in the nuclear power plant environment are required to address the possibility of troubles and malfunctions in instrumentation and control systems caused by electromagnetic emissions (EMI/RFI) from wireless technology. On the other hand, WLAN technology based on the IEEE 802.11 standards, has a very promising future for its use in nuclear power plants, due to its features like mobility, reliability, security, scalability and compatibility with other technologies. Currently, WLAN technology is been installing and evaluating in nuclear power plants worldwide, due to it provides enhanced features compared to traditional wireless technologies such as conventional mobile radio in two key aspects: higher operation frequencies and lower output power, which translates in very high data rates and 
very low electromagnetic interference. With regard to system design, a mobile IP telephony system based on wireless local area networks which will operate upon the existing CNLV's data backbone, has being proposed. In addition, the technical requirements that each commercially available component system must meet for its correct operation regarding the compliance with national and international standards, recommendations, regulatory guides, reliability and availability metrics, and security mechanisms, were established. Within the most important aspects identified in this work, are that the mobile IP telephony system must meet the design technical requirements for its exclusive operation in a nuclear power plant in Mexico, as well as to compliant to existing national and international standards applicable to nuclear power plants. Finally, the technical requirements of a network management system consisting of a network management server and network management software for the mobile IP telephony system, have been specified.

\section{References}

Shankar, R. (2003). Guidelines for Wireless Technology in Nuclear Power Plants, 11th International Conference on Nuclear Engineering, ICONE11, pp. 1-9, Tokio, Japan.

IEEE (1999a). IEEE Standard 802.11, Part 11: Wireless LAN Medium Access Control (MAC) and Physical Layer (PHY) Specifications.

IEEE (1999b). IEEE Standard 802.11b, Part 11: Wireless LAN Medium Access Control (MAC) and Physical Layer (PHY) Specifications. Higher Speed in the Physical Layer Extension in the $2.4 \mathrm{GHz}$ Band.

Martínez, E. (2002), Estándares de WLAN, Revista Red, No. 139, pp. 12-16, Mexico.

IEEE (1999c). IEEE Standard 802.11a, Part 11: Wireless LAN Medium Access Control (MAC) and Physical Layer (PHY) Specifications. High Speed Physical Layer in the $5 \mathrm{GHz}$ Band.

IEEE (2003). IEEE Standard 802.11g, Part 11: Wireless LAN Medium Access Control (MAC) and Physical Layer (PHY) Specifications. Further Higher Data Rate Extension in the $2.4 \mathrm{GHz}$ Band.

IEEE (2005). IEEE Standard 802.11e, Part 11: Wireless LAN Medium Access Control (MAC) and Physical Layer (PHY) Specifications. MAC enhancements for Quality of Service.

IEEE (2004). IEEE Standard 802.11i, Part 11: Wireless LAN Medium Access Control (MAC) and Physical Layer (PHY) Specifications. MAC enhancements for enhanced security.

NUREG (2003). Final report NUREG/CR-6782, Comparison of U.S. Military and International Electromagnetic Compatibility Guidance, USNRC, pp 34-36.

NRC (2003). RG 1.180, Guidelines for Evaluating Electromagnetic and Radiofrequency Interference in Safety-Related Instrumentation and Control Systems, U.S. Nuclear Regulatory Commission.

IEEE (1996). IEEE 1050 Standard, Guide for Instrumentation and Control Equipment Grounding in Generating Stations.

DoD (1999). MIL-STD-461e1 Standard, Requirements for the control of electromagnetic interference, characteristics of subsystems and equipment, U.S. Department of Defense.

IEC (2002). IEC 61000 Standard, Electromagnetic Compatibility (EMC)-Testing and Measurement Techniques, International Electrotechnical Committee.

EPRI (2003). Electric Power Research Institute (EPRI), EMI/RFI Issues, Technical Note, sections 3.3-3.6, pp. 49-50.

EPRI (2002). Electric Power Research Institute (EPRI), EPRI Report TR-03T023027, Guidelines for Wireless Technology in Nuclear Power Plants, available from 
http:/ / www.epri.com/targethigh.asp?program=249866\&value=03T023027\&objid= 284710.

FCC (2004). CFR 47, Part 15, Radio frequency Devices, Federal Communications Commission.

CCNNT (2001). NOM-121-SCT1-94, Telecomunicaciones - Radiocomunicaciones - Sistemas de

Radiocomunicación que emplean la Técnica de Espectro Disperso, Comité Consultivo Nacional de Normalización en Telecomunicaciones.

Meel, J. (1999). Report, Spread Spectrum (SS) Introduction, De Nayer Instituut, Belgium, pp. 1-33.

DoE (2002). U.S. Department of Energy, Industrial Wireless Technology for the 21st Century, white paper, DoE.

Pearce, J. (2001). FCC Considerations for Spread Spectrum Systems, available from http:/ / www.sss-mag.com/fccss.html.

Bahavnani, A. (2001). An Analysis of Implementing Wireless Technology to further enhanced Nuclear Power Plant Cost efficiency, Safety and Increased Employee Output, Pressure Vessel and Piping Design and Analysis, Vol. 430, pp. 369-372, ASME 2001.

Telrad Connegy (2001). Telrad Connegy web page, available from http://www.telradusa.com/pr_chernobyl.htm

Wireless Magazine (1995). Wireless Improves Safety at Hungary Nuclear Power Plant, Wireless Magazine, Vol. 4, No. 6, Nov/Dec, 1995.

EPRI (2004a). EPRI Wireless Technology newsletter No. 1009624, July 2004.

EPRI (2004b). EPRI Journal on-line, http://www.epri.com/journal/details.asp?doctype=products\&id=533\&flag=archi ve.

SpectraLink (2004). SpectraLink web page, available from http://www.spectralink.com/solutions/case.html

Kjesbu, S. and Brunsvik, T. (2000). Radiowave propagation in Industrial Environments, 26th Annual Conference of the IEEE Electronics Society, IECON 2000, pp. 2425-2430, Nagoya Japan.

IEEE (2002). IEEE 802.3 Standard, Local and Metropolitan Area Networks - Information Technology - Telecommunications and Information Exchange Between Systems - Local and Metropolitan Area Networks - Specific Requirements - Part 3: Carrier Sense Multiple Access with Collision Detection (CSMA/CD) Access Method and Physical Layer Specifications, Institute of Electrical and Electronic Engineers.

ITU (2009). H.323 Recommendation, Packet-Based Multimedia Communications Systems, International Telecommunications Union.

IETF (2002). RFC 3261, Session Initiation Protocol (SIP), Internet Engineering Task Force.

ITU (2005). H.248.1 Recommendation, Gateway Control Protocol, International Telecommunications Union.

ITU (1988). G.711 Recommendation, Pulse Code Modulation of Voice Frequencies, International Telecommunications Union.

ITU (2006). G.723.1 Recommendation, Dual Rate Speech Coder for Multimedia Communications Transmitting at 5.3 and $6.3 \mathrm{kbps}$, International Telecommunications Union.

ITU (2007). G.729 Recommendation, Coding of Speech at 8 kbps using Conjugate-Structure Algebraic Code Excited Linear-Prediction, International Telecommunications Union.

ITU (2009). G.168, Digital Network Echo Cancellers, International Telecommunications Union. IEC (2005). IEC 60950 Standard, Information Technology Equipment-Safety, International Electrotechnical Commission. 
IEEE (2003). IEEE 802.3af Standard, Power over Ethernet, Institute of Electrical and Electronic Engineers. 


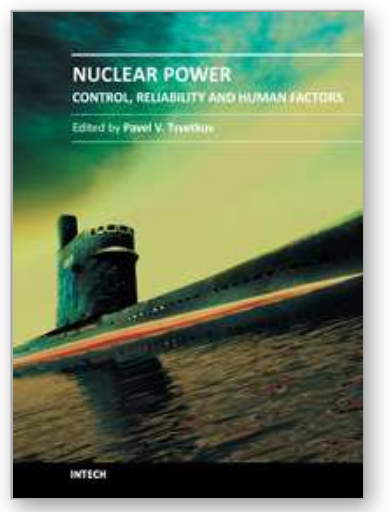

\author{
Nuclear Power - Control, Reliability and Human Factors \\ Edited by Dr. Pavel Tsvetkov
}

ISBN 978-953-307-599-0

Hard cover, 428 pages

Publisher InTech

Published online 26, September, 2011

Published in print edition September, 2011

Advances in reactor designs, materials and human-machine interfaces guarantee safety and reliability of emerging reactor technologies, eliminating possibilities for high-consequence human errors as those which have occurred in the past. New instrumentation and control technologies based in digital systems, novel sensors and measurement approaches facilitate safety, reliability and economic competitiveness of nuclear power options. Autonomous operation scenarios are becoming increasingly popular to consider for small modular systems. This book belongs to a series of books on nuclear power published by InTech. It consists of four major sections and contains twenty-one chapters on topics from key subject areas pertinent to instrumentation and control, operation reliability, system aging and human-machine interfaces. The book targets a broad potential readership group - students, researchers and specialists in the field - who are interested in learning about nuclear power.

\title{
How to reference
}

In order to correctly reference this scholarly work, feel free to copy and paste the following:

J. García-Hernández, J. C. Velázquez- Hernández, C. F. García-Hernández and M. A. Vallejo-Alarcón (2011). Design Considerations for the Implementation of a Mobile IP Telephony System in a Nuclear Power Plant, Nuclear Power - Control, Reliability and Human Factors, Dr. Pavel Tsvetkov (Ed.), ISBN: 978-953-307-599-0, InTech, Available from: http://www.intechopen.com/books/nuclear-power-control-reliability-and-humanfactors/design-considerations-for-the-implementation-of-a-mobile-ip-telephony-system-in-a-nuclear-powerplan

\section{INTECH}

open science | open minds

\section{InTech Europe}

University Campus STeP Ri

Slavka Krautzeka 83/A

51000 Rijeka, Croatia

Phone: +385 (51) 770447

Fax: +385 (51) 686166

www.intechopen.com

\section{InTech China}

Unit 405, Office Block, Hotel Equatorial Shanghai

No.65, Yan An Road (West), Shanghai, 200040, China 中国上海市延安西路65号上海国际贵都大饭店办公楼405单元

Phone: +86-21-62489820

Fax: +86-21-62489821 
(C) 2011 The Author(s). Licensee IntechOpen. This chapter is distributed under the terms of the Creative Commons Attribution-NonCommercialShareAlike-3.0 License, which permits use, distribution and reproduction for non-commercial purposes, provided the original is properly cited and derivative works building on this content are distributed under the same license. 